\title{
ANÁLISIS DE CONTENIDOS Y TENDENCIAS DE LAS WEBS DE BIBLIOTECAS ESCOLARES ANDALUZAS Y EXTREMEÑAS
}

\author{
Concepción María Jiménez Fernández* \\ UNIVERSIDAD INTERNACIONAL DE LA RIOJA
}

\begin{abstract}
Resumen: En este artículo se describe la investigación realizada a partir del análisis de webs de bibliotecas escolares de Andalucía y Extremadura con el fin de establecer un diagnóstico de la situación en cuanto a su valor pedagógico, formativo e informativo. Se describen los objetivos así como la metodología que se ha seguido para la realización del estudio y las conclusiones a las que se ha llegado tras analizar las 517 webs que forman la muestra aquí estudiada. Se termina con una propuesta para diseñar una web ideal de biblioteca escolar.

Palabras clave: bibliotecas escolares; web de biblioteca escolar; acceso a la información a través de la web; contenidos formativos en la web; aspectos pedagógicos de la web; Andalucía; Extremadura.
\end{abstract}

Title: CONTENTS AND TRENDS ANALYSIS OF ANDALUSIAN AND ESTREMADURAN SCHOOL LIBRARIES WEBSITES.

Abstract: In this article it is described the investigation carried out from the analysis of school libraries webs of Andalusia and Estremadura in order to establish a diagnosis of the situation as for his pedagogic, formative and informative value. There are described the aims as well as the methodology followed for the accomplishment of the study, and the conclusions obtained after analyzing 517 webs that form the sample here studied. It finishes with a proposal to design an ideal school library web.

Keywords: school libraries; school library web; access to information via web; training content on the web; pedagogical aspects of web; Andalusia; Estremadura.

\section{INTRODUCCIÓN.}

En el Manifiesto UNESCO/IFLA sobre la Biblioteca Escolar (2000) se señala que "la biblioteca escolar proporciona información e ideas que son fundamentales para desenvolverse con éxito en nuestra sociedad contemporánea, basada en la información y el conocimiento". La biblioteca escolar asume así un rol principal en el proceso de enseñanza y aprendizaje dentro de la escuela apoyando, entre otras cosas, la adquisición de nuevas competencias informacionales en el alumnado. Ese rol se ha ido incrementando gracias a la influencia de las nuevas corrientes pedagógicas, a las nuevas metodologías y enfoques curriculares y, también, gracias a la implementación de las nuevas tecnologías, concretamente internet ${ }^{1}$.

*concepcionm.jimenez@unir.net

Recibido: 10/10/2012; 2a versión: 29/11/2012; 3ª versión: 15/01/2013; aceptado: 07/02/2013.

JIMÉNEZ FERNÁNDEZ, C.M. Análisis de contenidos y tendencias de las webs de bibliotecas escolares andaluzas y extremeñas. Anales de Documentación, 2013, vol. 16, n ${ }^{\circ} 1$. Disponible en: http://dx.doi.org/10.6018/analesdoc.16.1.160221 
Aunque internet tuviese un origen militar ${ }^{2}$, ha llegado a convertirse en una herramienta que permite que todas las personas, de cualquier lugar del mundo, puedan comunicarse y participar. Ha supuesto, por tanto, un antes y un después en el intercambio de información. Esto implica que el uso de la red no solo tenga un objetivo profesional sino de recreo, de compartir intereses comunes, interactuar con usuarios afines. Todo en igualdad de condiciones. Además, la rapidez y la inmediata difusión de la información a través de la red, facilita el establecimiento de proyectos educativos comunes eliminando barreras geográficas y sociales. Para el alumnado, internet es una importante fuente de aprendizaje y de comunicación.

En ese sentido, y en relación con el uso de internet en educación, Romagnoli C., Femenías, G. y Conte, P. (2001 p. 34), afirman que ese uso se lleva a cabo con diferentes miras:

- Estimular el uso de nuevas formas de aprender y de construir el conocimiento

- Facilitar el aprendizaje de otros y con otros

- Estimular destrezas sociales y cognitivas

- Aprender haciendo, construyendo cosas y resolviendo problemas

- Aportar nuevas herramientas de apoyo a la realización de trabajos colaborativos

- Estimular el trabajo global e interdisciplinario

Sin embargo, esta categorización carece de una alusión a la utilización de la red para acceder a cualquier información que se necesite pero de manera eficiente. Está claro que en internet se puede encontrar información en diferentes formatos como imagen, texto o sonido. Pero esta información, y cualquier otra en general, no es conocimiento. En educación, como en otros ámbitos, se necesita aprender no solo a buscar información sino a analizarla, filtrarla, criticarla, organizarla y transformarla en conocimiento, es decir, dotar a los alumnos de habilidades básicas relacionadas con la alfabetización en información. Y esto se hace posible desde la biblioteca escolar, lugar donde se albergan los recursos tanto impresos como digitales.

Pero si al hablar de internet se hace alusión a la red física que une ordenadores, la aplicación a través de la cual los usuarios pueden intercambiar información es la Web. En este sentido, Castells (2001, p. 29) afirma que "lo que hizo posible que internet abarcara todo el planeta fue el World Wide Web. Esta es una aplicación para compartir información desarrollada por Tim Berners-Lee en 1990".

Berners-Lee desarrolló un sistema, la web, que según sus propias palabras “es más una creación social que técnica. Yo lo diseñé por su efecto social -para ayudar a que la gente trabajase junta- y no como un juguete técnico. El objetivo último de la web es apoyar y mejorar nuestra entretejida existencia en el mundo" (Berners-Lee, 2000, p. 115). 
Ese desarrollo de la Web ha supuesto un gran avance también en las bibliotecas escolares puesto que, poco a poco, han ido aprovechando el potencial que el nuevo entorno les brinda para organizar y difundir su trabajo en el centro educativo. La Web se convierte de este modo en un recurso imprescindible en la imagen de la biblioteca escolar, "porque la web contiene y proyecta, en cierta manera como un gran espejo, lo que somos, para bien y para mal" (García Andrés 2008, p. 24). La web de la biblioteca escolar es por tanto un escaparate y una manera de ofrecer una serie de servicios y productos de la propia biblioteca. Por ello, entre sus objetivos está el facilitar a los usuarios el acceso a la información que se ofrece a toda la comunidad escolar.

\section{OBJETIVOS Y METODOLOGÍA.}

El objeto de la presente investigación es analizar el valor informativo y pedagógico de las webs de bibliotecas escolares de centros educativos de primaria en Andalucía y Extremadura y es que, como dice Yáguez, "elaborar la web de la biblioteca debería ser uno de los objetivos, si no a corto plazo, al menos a largo plazo en la era de las nuevas tecnologías" (Yáguez, 2006, p. 95). Además, se aportan una serie de pautas que servirán para crear una web modelo de biblioteca escolar. Esa web modelo, aunque pueda parecer poco realista, es una meta a la que hay que tender y cuyo objetivo principal sería responder a necesidades formativas e informativas del alumnado de educación primaria. No obstante, el estudio de esas necesidades no se incluye en este trabajo debido al formato reducido del mismo, pero sí supone un campo de estudio complementario del análisis de los contenidos de las webs de biblioteca escolar de primaria.

Además, a través de este estudio, otras administraciones educativas -además de las dos aquí estudiadas- podrían estar interesadas en conocer los resultados obtenidos como una aproximación a la realidad de las webs de sus bibliotecas escolares de educación primaria, así como las propuestas que se aportan para poder mejorar esa realidad. Se pretende que sirva también como herramienta de diagnóstico para que dichas instituciones educativas dispongan de unas pautas para la creación de contenidos web de utilidad para las bibliotecas escolares.

En la realización de este trabajo, se han tomado como referencia dos comunidades autónomas cercanas geográficamente -Andalucía y Extremadura- y con una característica común, la de ser pioneras en el uso de las TIC en Educación (Cervero Guerrero, 2009). Por otra parte, se ha decidido estudiar las webs de bibliotecas escolares de los centros de primaria, ya que se considera que es justo en esa etapa donde la biblioteca se convierte en el espacio en torno al cual se movilizan las diferentes competencias básicas del alumno antes de que este llegue a secundaria (España, 2006).

También era imprescindible conocer no solo el número de centros de educación primaria existentes en las dos comunidades autónomas sino cuántos de esos centros contaban con página web y cuántos tenían página web de biblioteca escolar. Tras un minucioso rastreo y consulta de diferentes fuentes de información institucionales y 
personales -tanto de ámbito nacional como regional y provincial-, se comprobó que el número total de centros de primaria en las dos comunidades es de 3.042 , de los cuales 2.125 poseen web de centro y 517 cuentan con web de biblioteca escolar. Desglosando este último dato por comunidades autónomas, se aprecia que 419 webs de biblioteca escolar -de las 517 existentes- corresponden a Andalucía y 98 a Extremadura (tabla I). Para la realización del análisis se ha establecido como muestra de estudio las 517 webs de biblioteca escolar, es decir, el cien por cien de las webs existentes en las dos comunidades autónomas analizadas.

\begin{tabular}{|c|c|c|c|}
\cline { 2 - 4 } \multicolumn{1}{c|}{} & Número de centros & Cetros con web & $\begin{array}{c}\text { Con web de } \\
\text { biblioteca escolar }\end{array}$ \\
\hline Andalucía & 2558 & 1662 & 419 \\
\hline Extremadura & 484 & 463 & 98 \\
\hline TOTAL & 3042 & 2125 & $\mathbf{5 1 7}$ \\
\hline
\end{tabular}

Tabla I. Número de centros educativos de primaria de Andalucía y Extremadura, con web de centro y con web de biblioteca escolar.

Para valorar los contenidos relacionados con la información pedagógica, formativa e informativa que presenta cada una de las 517 webs de bibliotecas escolares, era necesario establecer un esquema de evaluación. Tras la lectura de diferentes propuestas de distintos expertos en la materia (García Guerrero y Luque Jaime, 2011b; Jiménez Piano y OrtizRepiso Jiménez, 2007; Murphy, 2003), se diseñó un patrón de medida propio. Dicho patrón se compone de una serie de variables relacionadas con la navegación a través de la web, la información sobre la biblioteca, actividades de promoción de la lectura, apoyo a la docencia, acceso al catálogo, información y referencia, actualización de la web e interacción con los usuarios. Se han considerado una serie de aspectos o elementos que deberían estar presentes de una manera u otra en las webs de biblioteca escolar. El esquema considera las siguientes variables:

1. Enlaces que faciliten la navegación:

- Vínculos bien estructurados, etiquetados, claros y precisos

2. Información sobre la biblioteca:

- Plano

- Horarios

- Responsable

- Reglamento de la biblioteca

- Normativa de préstamo

3. Actividades de promoción de la lectura:

- Estrategias que sirvan para afianzar hábitos lectores 
4. Materiales de apoyo a la docencia:

- Recursos vinculados a la actividad formativa e informativa de interés para la docencia

5. Acceso al catálogo de la biblioteca:

- Información sobre el contenido de la colección y su ubicación

6. Servicios de información y referencia:

- Referencia

- Orientación bibliográfica

- Enlaces externos

- Libros electrónicos

- Otros enlaces a recursos

7. Actualización de la web durante el curso escolar 2010/2011:

- Indicaciones sobre su actualización durante el curso académico 2010/2011

8. Posibilidad de interacción con el usuario:

- Correo electrónico de la biblioteca o del coordinador

- Teléfono de la biblioteca

- Aplicaciones de la web 2.0

- Formulario de contacto

- Desiderata en línea

De la aplicación del modelo descrito a cada web analizada se obtiene un valor. Este valor se aplica según la presencia o no en la web de alguno de los aspectos analizados en cada variable. No se ha valorado en qué medida esos aspectos están o no presentes en la web -tema que puede ser objeto de un estudio posterior- sino si esos aspectos están o no presentes en la web para, posteriormente, proporcionar un modelo de web ideal de biblioteca escolar. 


\section{RESULTADOS}

Tras aplicar el modelo de análisis diseñado, se obtienen datos concretos (figura 1) y una serie de resultados asociados sobre el cumplimiento de las distintas variables ${ }^{3}$ en las webs de bibliotecas escolares de los centros de educación primaria de Andalucía y Extremadura.

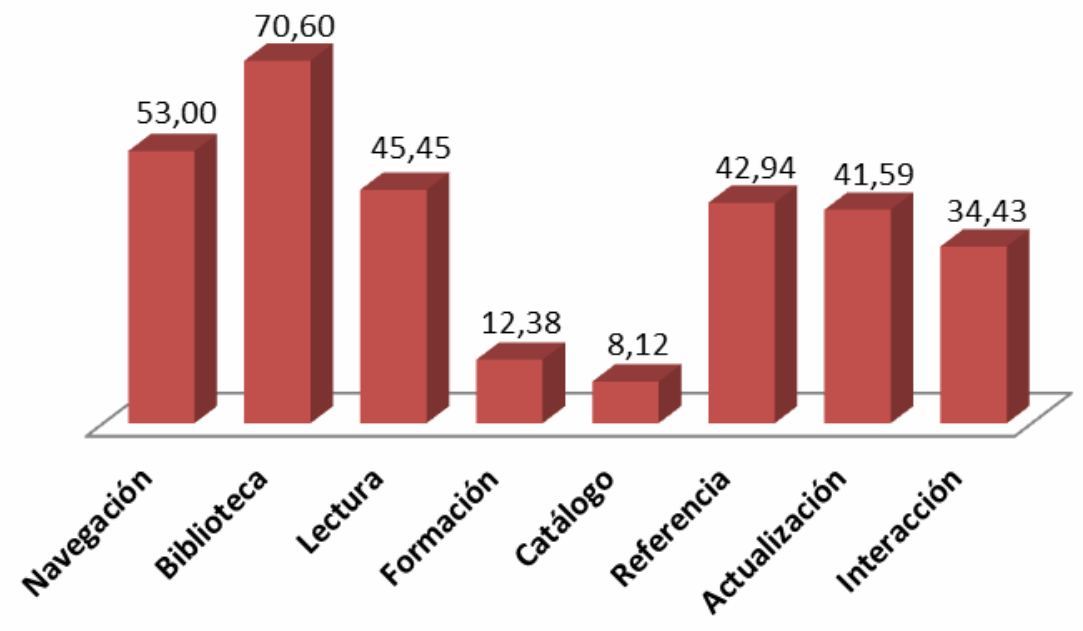

Figura 1. Datos globales porcentauales del cumplimiento de cada una de las variables en Andalucía y Extremadura.

De las 517 webs de biblioteca escolar de Andalucía y Extremadura, se aprecia que la variable que más destaca sobre las demás es la correspondiente a la inclusión de algún tipo de información sobre la biblioteca con un 70,60\%. Por el contrario, los datos más preocupantes son los correspondientes a la posibilidad de acceso al catálogo desde la propia página, con un $8,12 \%$.

Según los datos obtenidos en el estudio, resulta inquietante que sean menos de un diez por ciento las webs de biblioteca escolar que aporten datos sobre el contenido de sus colecciones como una puerta más de entrada a cualquier biblioteca. Con estos resultados queda patente que la gran mayoría de las webs de biblioteca escolar de los centros educativos de primaria no parecen tener interés en mostrar a sus usuarios los materiales que contiene la biblioteca -ya sea a través de un catálogo en línea o a través de un listado de esos materiales consultable a través de esa web- y con los que se pueden satisfacer las necesidades formativas, informativas y de recreo. La razón puede estar justificada por la 
facilidad de acceso a la biblioteca física al estar ésta integrada en el centro y a la que se puede acudir a diario de forma presencial.

Otro aspecto destacable es el bajo porcentaje de webs que facilitan materiales y actividades de apoyo a la docencia y la formación (12,38 \%). Este resultado no es el esperado puesto que siendo la biblioteca escolar un eje esencial para el desarrollo del proceso de enseñanza-aprendizaje en la escuela. Resulta extraño que solo sean poco más del diez por ciento las webs de bibliotecas escolares que proporcionan estos materiales que servirían como fuente de información y apoyo a la labor docente. No obstante, se ha de tener en consideración, en este aspecto y en el resto de los estudiados aquí, que el centro puede disponer de una intranet que ofrezca determinados contenidos y servicios al alumnado y al profesorado, pero que no aparecen en la web general de la biblioteca escolar.

En lo referido a la variable Lectura, y al igual que ocurre con los datos obtenidos de las variables Referencia, Actualización e Interacción -todas ellas en un nivel intermedio-, no aparecen desigualdades relevantes entre sus valores porcentuales si bien hay que destacar que ninguna de estas cuatro variables supera el $50 \%$. Detallando un poco más estos resultados, se puede apreciar, según los datos obtenidos, que la variable Lectura y la variable Referencia cuentan con porcentajes muy similares. Estos resultados llaman la atención puesto que parece previsible que en la biblioteca escolar de un centro de primaria se difundan más las actividades de promoción de la lectura que realizan -dando así protagonismo y participación a los alumnos ${ }^{4}$ - que los servicios de información y referencia, más utilizados en otro tipo de bibliotecas como podrían ser las universitarias, con un público con necesidades más amplias, diversas y especializadas.

La actualización de la web resulta ser otro de los aspectos que cuenta con una valoración por debajo del $50 \%$. Según los resultados del análisis, se aprecia que menos de la mitad de las webs de bibliotecas escolares no muestran ningún dato que indique la fecha de la última modificación o inserción de contenidos nuevos. Este aspecto es destacable puesto que la no inclusión de la última actualización podría provocar confusión entre los usuarios que visitan la página, al no saber a qué fecha corresponde la información o los contenidos de esa web.

También queda de manifiesto que en el $34,43 \%$ de estas páginas no existe la posibilidad de interactuar o relacionarse con la persona que la visita, ni por medio del correo electrónico, el teléfono o cualquier otro sistema de comunicación. Se ha de tener en cuenta que la interacción es esencial para la retroalimentación con el usuario y su participación en la vida de la biblioteca.

En un nivel superior al cincuenta por ciento se encuentran la variable Navegación y la variable Biblioteca. Se percibe, según los resultados, una preocupación por parte de las bibliotecas escolares por facilitar la forma de navegar o estructurar su web para lograr hacerla más accesible y clara a sus usuarios. 
Pasando a desglosar los datos por comunidades autónomas, se considera interesante estudiar los resultados globales en cada una de ellas así como los resultados según las diferentes variables en cada una de las dos comunidades.

Del total de centros educativos con web de biblioteca escolar analizados, y como se puede apreciar en la Figura 2, la mayoría corresponde a Extremadura con un 20,25\% frente a un 16,38 \% de centros con web de biblioteca escolar que corresponden a Andalucía.

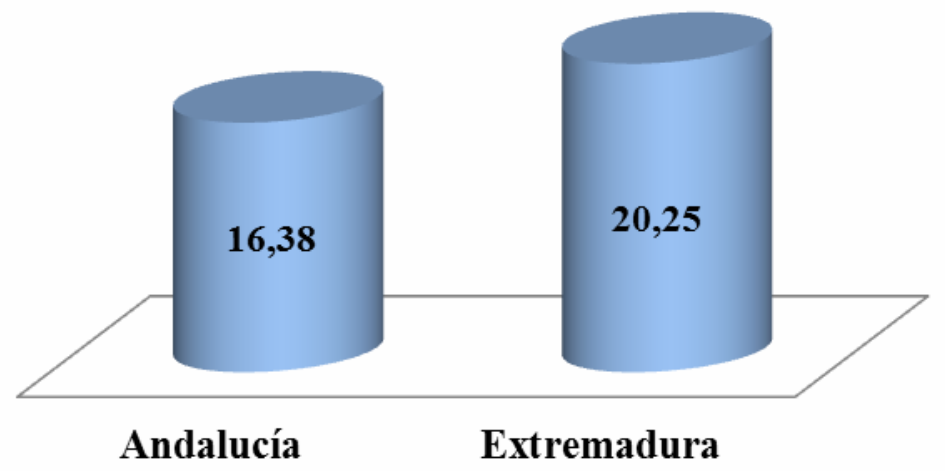

Figura 2. Porcentaje de centros educativos con web de biblioteca escolar en Andalucía y Extremadura.

Este dato es significativo si se tiene en cuenta que la comunidad autónoma de Andalucía está compuesta por ocho provincias y, además, posee muchos más centros educativos de educación primaria -en concreto 2.558 centros- que la comunidad autónoma de Extremadura, que solo cuenta con dos provincias y con un número de centros educativos de primaria mucho más reducido -484 centros educativos.

En la figura 3 se representan los resultados obtenidos en el análisis de las ocho variables, diferenciándolas según cada comunidad autónoma. Tanto Andalucía como Extremadura cuentan con valores porcentuales muy similares en la mayoría de las variables estudiadas. Observándo la figura con más detalle, se aprecia que en más de la mitad de las webs se percibe una preocupación por presentar los contenidos de forma estructurada y claramente señalizada, lo que facilita la navegación. Se ha de volver a señalar que es la variable Biblioteca la que destaca sobre todas las demás con un mayor valor porcentual tanto en webs de biblioteca escolar de Andalucía como en Extremadura lo que se traduce en que la gran mayoría de las bibliotecas escolares de las dos comunidades autónomas consideran de gran importancia dar a conocer sus servicios y funcionamiento a todo aquel que visite la web, aunque, como ya se ha comentado, puede 
ocurrir que la web no necesariamente exponga sus contenidos públicamente sino que lo haga de manera interna, a nivel interno.

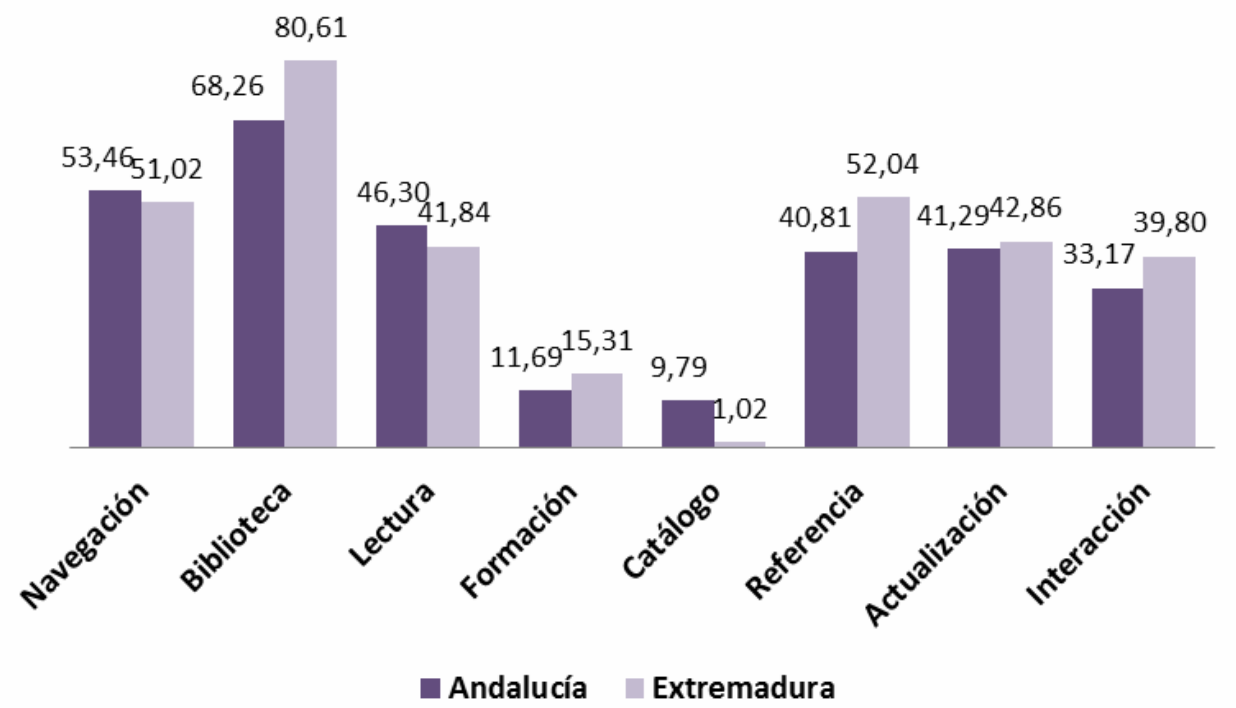

Figura 3. Porcentajes totales según cada variable en Andalucía y en Extremadura.

Según los datos, se aprecia que en ninguna de las dos comunidades autónomas se perciben diferencias significativas en lo que se refiere a resultados de la variable Lectura. Este dato es preocupante ya que ninguna de las dos comunidades estudiadas supera el cincuenta por ciento de webs que ofrezcan información sobre las actividades de promoción de la lectura que realizan. La explicación puede ser la falta de coordinación entre biblioteca y trabajo en el aula -lo que implica que desde la biblioteca no se conozca lo que se está realizando en clase para fomentar la lectura de los alumnos-, o bien por la posible falta de conocimientos informáticos o de tiempo de la persona encargada de mantener la web a la hora de publicar imágenes o vídeos sobre las actividades de animación lectora que se organizan desde la biblioteca.

Igual de parecido -y teniendo en cuenta que nos referimos a la información pública en la web de biblioteca escolar-, es el resultado de la variable Formación, materiales o actividades de apoyo a la docencia y formación. Como puede observarse, los centros educativos de primaria de las dos comunidades no parecen considerar este aspecto como uno de los más relevantes a tener en cuenta a la hora de incluir esta información en las webs de biblioteca escolar. De nuevo, la explicación puede venir dada por la falta de coordinación entre la biblioteca y el profesorado para conocer cuáles son los contenidos curriculares que se imparten en el aula o bien por la inexistencia de personal dedicado en exclusiva a la gestión de contenidos digitales para el aula desde la biblioteca. 
Especialmente relevante es el resultado correspondiente a la variable Catálogo. Se ha de indicar que esta variable, es la que destaca sobre todas las demás con el valor porcentual más bajo en las webs de bibliotecas escolares de centros de educación primaria.

En cuanto a los servicios de información y referencia se aprecia que son menos de la mitad de las webs analizadas las que ofrecen este servicio a sus usuarios, mientras que en la comunidad extremeña son poco más de la mitad las que sí lo proporcionan. Es evidente que en las dos comunidades autónomas se otorga una importancia intermedia a este aspecto. Llama la atención que los servicios de información y referencia -mucho más utilizados en otras bibliotecas de centros de enseñanza como las universitarias, donde los usuarios requieren una información más elaborada- estén presentes en la mitad de las webs de biblioteca escolar de centros educativos de primaria.

En cuanto a la actualización de la web, ninguna de las dos comunidades autónomas llega al $50 \%$. Este dato pone de relevancia que menos de la mitad de las bibliotecas escolares de primaria de las dos comunidades autónomas aporta información sobre cuándo ha sido la última vez que amplió o modificó los contenidos de la web. El resultado es significativo porque en bibliotecas escolares, donde pueden existir cambios relevantes de un curso académico a otro, la indicación de la fecha de actualización es esencial para conocer a qué curso se refiere la información contenida en la web: horarios de la biblioteca, actividades de promoción lectora, etc. Esto puede ser debido a que la persona encargada de publicar los contenidos a la web puede dar por hecho que los únicos usuarios que acceden a ella son miembros de la comunidad escolar y dar por hecho también que esos usuarios conocen qué se está haciendo desde la biblioteca en ese curso académico por lo que no consideran importante añadir la fecha de actualización.

Por último, queda de manifiesto que la mayoría de las webs de biblioteca escolar de educación primaria de Andalucía y Extremadura no conceden demasiada importancia a la comunicación y relación con sus usuarios vía web. La causa puede estar en que esos aspectos -la comunicación y relación con los usuarios- ya estén suficientemente asegurados y consolidados en su forma presencial y no se requiera de otro canal adicional que exija un mayor esfuerzo de atención y dedicación, por lo que no compensa su puesta en marcha y mantenimiento.

\section{ANÁLISIS DE RESULTADOS.}

Consideramos que, tras el análisis de los resultados, se obtiene una estimación fiable de la situación real de las webs de las bibliotecas escolares de primaria en Andalucía y Extremadura en lo que concierne a esta información pública.

Se puede afirmar que en las dos comunidades autónomas estudiadas el resultado es similar, por lo que simulando las calificaciones escolares - de muy deficiente a 
sobresaliente- se puede apreciar la distancia existente entre la puntuación obtenida en cada una de las variables analizadas de forma general y la puntuación máxima posible considerada (10).

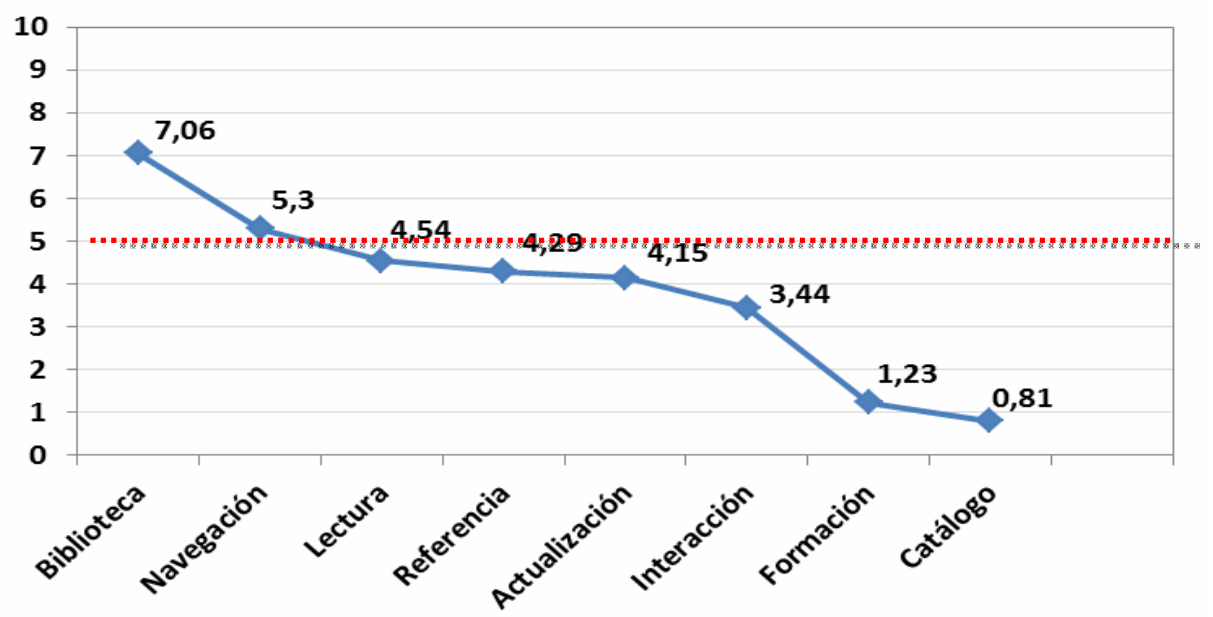

Figura 4. Distancia existente entre valor ideal y el valor real de cada variable.

Los valores más próximos al sobresaliente -el máximo- son los relacionados con la información sobre la biblioteca y los enlaces que facilitan la navegación -con notable y suficiente respectivamente-. Por debajo del cinco, con un insuficiente, estarían los aspectos relacionados con las actividades de promoción de la lectura, información y referencia, actualización de la web e interacción con el usuario. Con un muy deficiente estarían los materiales de apoyo a la docencia -recursos vinculados a la actividad formativa e informativa de interés para el docente- y el acceso al catálogo a través de la web.

La información sobre cómo es y cómo funciona la biblioteca es la que más presencia tiene en las webs de bibliotecas escolares. El especial alcance que permite internet convierte a las webs en un soporte fundamental para la exposición de ese tipo de información. Esta información sobre la biblioteca era de esperar que obtuviera la valoración más alta puesto que toda web es el escaparate de cualquier institución o empresa. En el caso de las bibliotecas escolares se aprecia que tienen gran interés en darse a conocer a través de su web mostrando cuáles son sus instalaciones, horarios, reglamentos y normativa de préstamo. Se valora positivamente el esfuerzo que hacen las bibliotecas para ofrecer esos datos como "tarjeta de presentación" ya que se entiende que es importante que la web de la biblioteca escolar facilite esta información al usuario sin necesidad de tener que acudir físicamente a ella.

No se da la suficiente importancia a las actividades de promoción de la lectura-que se queda ligeramente por debajo del aprobado-, los servicios de información y referencia o 
la actualización de la información. Lo ideal sería que todas las webs posibilitasen el acceso a dichas actividades, puesto que estas mejoran los hábitos lectores de los alumnos así como su calidad y comprensión lectora, algo esencial para entender cualquier texto. Además, estas actividades sirven para dar a conocer los fondos y materiales con los que cuenta la biblioteca e introducen al alumno en el mundo de la literatura estimulando sus deseos de comunicarse y expresarse. Pero, puede ser que no se lleven a cabo este tipo de actividades debido al tiempo escaso del que se dispone en la biblioteca escolar, dándole más importancia a otras labores como la catalogación y la clasificación de los fondos. También podría ocurrir que las actividades de promoción de la lectura que se llevan a cabo tengan un carácter no colectivo (implicando a todos los alumnos de un determinado nivel) sino que son realizadas en clase, de forma aislada, por un determinado profesor de una asignatura concreta, generalmente el profesor de Lengua y Literatura. Por este motivo es posible que no se considere adecuada para una difusión general, o que el profesor que la lleva a cabo no tenga relación con la biblioteca y sus servicios por falta de un plan integrado de lectura coordinado con la biblioteca.

Se echa de menos en la mayoría de las webs el servicio de referencia y de orientación bibliográfica. Son pocas las bibliotecas que cuentan con este servicio a través de sus páginas webs, pero esta ausencia puede venir justificada por el tipo de biblioteca que se está analizando. Es una característica muy generalizada que la biblioteca escolar no cuente con un bibliotecario profesional o dedicado a tiempo completo sino que suele ser un maestro el que dedique algunas horas a la atención de la misma y sus usuarios. Este podría ser uno de los motivos por los que el servicio de referencia digital -al igual que otros muchos aspectos analizados en este estudio- no se suele encontrar en las webs de biblioteca escolar.

La importancia de la actualización de la web no es nimia ya que unos contenidos desfasados u obsoletos provocan la desconfianza y el rechazo del usuario mientras que la actualización contribuye a mantener su interés y a revisitar la web.

Este dato es preocupante puesto que una de las características de la web es la interacción con el usuario. Plasmar contenidos en una web sin ofrecer la posibilidad de interactuar con el usuario mediante correo electrónico, teléfono, formulario o cualquier otro medio es hablar de web 1.0, una web estática, sin atisbo de dinamismo y sin intención de hacer miembros y partícipes a sus usuarios en la vida de la biblioteca desde cualquier parte y en cualquier momento. El motivo puede explicarse por la posible suposición -por parte de la persona encargada de la web- de que si el usuario necesita alguna información o quiere realizar alguna consulta acudirá físicamente a la biblioteca, lo cual no es descartable en un espacio ubicado en el mismo edificio al que acuden a diario, la escuela.

La inexistencia del catálogo, una forma más de facilitar el acceso a los contenidos tanto bibliográficos como no bibliográficos que la institución contiene y pone a disposición de sus usuarios -además del libre acceso a los fondos-, puede venir explicada por el esfuerzo y dedicación que supone no solo su confección sino también su 
mantenimiento y actualización, algo difícil de conseguir en bibliotecas donde no suele haber un bibliotecario dedicado a tiempo completo.

La posible causa del bajo porcentaje en la variable Formación puede ser la falta de un plan de lectura y biblioteca en el centro con el que se puede lograr un consenso entre la biblioteca y las necesidades curriculares en el aula. La falta de coordinación entre biblioteca y profesorado para conocer cuáles son los contenidos que se imparten en clase puede explicar esta situación.

Por otra parte, únicamente se suele asociar el contenido de la biblioteca escolar con el área de Lengua y Literatura, por lo que muchos profesores no son conscientes de las posibilidades que una biblioteca bien gestionada y organizada puede ofrecerles para el desarrollo de su trabajo. Por lo general, al frente de la biblioteca escolar suele estar un profesor que cubre de esta forma sus horas libres o que solo la abre durante el recreo para que los niños que voluntariamente quieran ir puedan sacar un libro en préstamo. Como ya se ha comentado, la inexistencia de un bibliotecario a tiempo completo y preocupado por satisfacer las necesidades informativas, formativas y de recreo de sus usuarios implica, entre otras muchas cosas, que no se puedan aportar estos materiales de apoyo a los profesores de las distintas áreas.

Aunque a la mayoría de las variables les queda un amplio trecho para alcanzar el máximo -el diez-, las dos más bajas, muy cercanas a cero, deberían hacer un esfuerzo mayor para aproximarse al extremo superior.

\section{CONCLUSIONES: HACIA UNA PROPUESTA DE WEB PARA UNA BIBLIOTECA ESCOLAR DE EDUCACIÓN PRIMARIA.}

Este estudio, además de presentar un diagnóstico de la situación de las webs de biblioteca escolar en Andalucía y Extremadura, pretende aportar un modelo de web útil y aplicable a cualquier centro de primaria. Para ello se expone una propuesta cuyos contenidos quedan agrupados según las ocho variables estudiadas:

1. Herramientas de navegación

2. Información de la biblioteca

3. Promoción de la lectura

4. Apoyo a la formación

5. Acceso al catálogo

6. Información y referencia

7. Información actualizada

8. Recursos de información y relación

Los distintos apartados de esta propuesta ideal están basados en el estudio realizado y posterior análisis de resultados. Si se decidiese salir de las dos comunidades autónomas estudiadas -Extremadura y Andalucía- se observa que la propuesta es extrapolable a 
cualquier comunidad española por la muestra tan amplia que se ha manejado para la realización de este estudio.

La web ideal incluiría, por tanto, y de manera resumida, los siguientes puntos clave:

\section{Herramientas de navegación}

La facilidad de navegación es fundamental para lograr una funcionalidad educativa de la web. Así, se ha de facilitar al máximo el desplazamiento y la navegación del usuario en la web. Para ello se tienen que ofrecer enlaces claros que sirvan para organizar y localizar de forma sencilla y rápida la información que la web contiene o para dar a conocer el lugar de la web donde se encuentra en cada momento.

Se considera importante no solo el contenido sino la forma de presentarlo, con palabras y frases sencillas -evitando ambigüedades-, destacando de algún modo la información más reciente o la más importante y con una distribución espacial del texto que facilite su lectura de forma ordenada. Se trata de pensar en digital para no convertir la web en una extensión de la guía impresa de la biblioteca.

\section{Información sobre la biblioteca}

Las webs de las bibliotecas escolares deberían ofrecer una información sobre la biblioteca como espacio de trabajo y servicio, sobre cómo y cuándo poder acceder a ella, a sus servicios y a sus recursos. Entre las posibles secciones relacionadas con este aspecto de la información sobre la biblioteca, se contemplan las siguientes: horario, descripción de los espacios, responsable de la biblioteca, reglamento y normas de uso.

En este sentido, sería interesante utilizar formatos multimedia-vídeos de presentación, imágenes fotográficas, etc.-, o herramientas de la web 2.0 que faciliten el acceso a esa información de manera más atractiva dándole así un valor añadido a la presentación de esos datos en la web.

\section{Promoción de la lectura}

La biblioteca escolar ha de ofrecer información sobre las actividades de promoción de la lectura que realiza, ha realizado o realizará en el futuro, encaminadas a afianzar hábitos de lectura y escritura de los alumnos. Para ello, las TIC se convierten en el mejor aliado con miras a organizarlas, ponerlas en marcha y difundirlas.

Estas actividades deben presentarse de manera diferenciada según los tramos de edad a los que van dirigidas, para así orientar sobre su desarrollo y objetivos a potenciales visitantes de la web o para informar a padres y familias sobre las estrategias de promoción de la lectura en las que sus hijos pueden participar, implicándolos a ellos también. 
No se puede olvidar la promoción lectora entre profesores. De este modo, desde la web de la biblioteca se podría crear un club de lectura virtual en el que pudieran participar todos aquellos profesores que lo deseen.

Todas las actividades deben realizarse en colaboración con el profesorado y han de formar parte del proyecto educativo del centro. Las actividades realizadas de manera aislada no suelen afianzar el gusto por la lectura.

\section{Apoyo a la docencia}

El libro de texto sigue siendo el preferido en clase desaprovechando otro tipo de información con la que se pueden desarrollar diferentes habilidades entre el alumnado y que puede ser suministrada por la biblioteca escolar.

La web debería contar con un espacio donde poder compartir información, materiales y recursos sobre temas relacionados con las áreas curriculares que se imparten en clase.

Es necesario, por tanto, integrar la biblioteca en todas las áreas curriculares y no solo en la de Lengua y Literatura como suele ser habitual.

Si la biblioteca escolar oferta este tipo de materiales a los profesores a través de su web, estos la percibirán como un verdadero centro de recursos que pueden utilizar para hacer más fácil su labor docente. Además, los alumnos, al comprobar que sus profesores utilizan los recursos que les ofrece la biblioteca se sentirán más motivados a utilizarla, tanto para el ocio como para sus necesidades informativas y de formación.

\section{Acceso al catálogo}

Ese acceso podría estar disponible en línea, junto con otros servicios asociados a las consultas en el catálogo como dudas, reservas, renovaciones, préstamo interbibliotecario, novedades, etc. En caso de ser una biblioteca con un menor volumen de fondos, enlaces a archivos en pdf o en cualquier otro formato que den cuenta del contenido y ubicación de esos fondos en la biblioteca. De la manera que sea, una biblioteca debería ofrecer este modo de acceso a la colección de la biblioteca.

\section{Información y referencia}

La web de la biblioteca escolar ha de incluir servicios de:

- Información y referencia que sirvan para responder a cuestiones diversas y ofrecer respuestas rápidas y concretas a cuestiones puntuales.

- Orientación bibliográfica, como un servicio de asesoramiento al usuario sobre dudas relacionadas con el fondo o sobre cómo y dónde buscar la información que 
necesita o cómo tratarla y evaluarla (mediante tutoriales, vídeos, presentaciones, etc.).

- Enlaces externos a otros recursos relacionados con la lectura y el libro o con posibles necesidades formativas e informativas de la comunidad educativa, traductores de textos o buscadores.

- Libros electrónicos (accesibles para su lectura en pantalla o bien a través de códigos QR con los que poder descárgarlos desde cualquier dispositivo móvil), acceso a prensa digital (para estar al día de la actualidad informativa) revistas electrónicas que puedan posibilitar su relación con los contenidos impartidos en clase.

- Otros enlaces relacionados con otras bibliotecas escolares o públicas con las que se pueda colaborar para lograr un mayor aprovechamiento de los recursos.

\section{Información actualizada}

La web de biblioteca escolar, como cualquier otra web, debe estar continuamente actualizada si se desea que esta sea útil y vigente. Actualizar significa añadir periódicamente nuevos contenidos y recursos que resulten útiles a los usuarios. Pero, tan importante es añadir contenidos nuevos como eliminar o modificar aquello que se encuentra desfasado $\mathrm{u}$ obsoleto.

Sería un fallo creer que cuando se ha diseñado una web esta ya está terminada. Una web es un espacio en continuo crecimiento y su construcción exige un compromiso para mantenerla, actualizarla y dinamizarla.

\section{Recursos de comunicación y relación}

Es necesario establecer un canal de comunicación permanente entre la biblioteca y el usuario ya que permite una retroalimentación que contribuye a detectar problemas; conocer inquietudes de los usuarios; establecer puntos de mejora a través de las sugerencias aportadas; formular consultas; etc. Para ello se ha de diseñar un espacio que posibilite la participación activa del usuario. De no hacerlo de esta manera, se seguirá desarrollando una formación en la que sigue primando el sujeto pasivo, es decir, el usuario no activo, una actividad semejante a la colocación de un folleto en el mostrador de entrada de la biblioteca solo que cambiando el canal de distribución.

Comunicación e interacción a través de la presencia de la biblioteca en las redes sociales, comunicación e interacción a través de dispositivos móviles como smartphones, blogs, sindicación de contenidos...

No cabe duda de que las tecnologías son un potenciador de utopías y sueños. Esos sueños, que en otros tiempos eran difíciles de alcanzar, ahora pueden hacerse realidad muy fácilmente gracias al uso de las tecnologías, por lo que sería un gran error desaprovechar esta oportunidad. Solo hay que querer. 


\section{NOTAS.}

\footnotetext{
${ }^{1}$ A lo largo de este trabajo se utilizará el término internet en minúscula tal y como se refleja en el avance de la vigésimo tercera edición del Diccionario de la lengua española, Real Academia Española.

${ }^{2}$ Internet tiene su origen en el proyecto Arpanet (1968), en Estados Unidos. La idea original residía en construir una red capaz de transportar información de ámbito militar y gubernamental.

${ }^{3}$ Con la intención de facilitar la lectura de algunas de las figuras aquí incluidas, se ha resumido cada una de las variables en solo una palabra que identifique su contenido. De este modo las variables quedan sintetizadas de la siguiente manera: 1. Navegación, 2. Biblioteca, 3. Lectura, 4. Formación, 5. Catálogo, 6. Referencia, 7. Actualización y 8 . Interacción.

${ }^{4} \mathrm{Se}$ ha de recordar que los alumnos de primaria tienen edades comprendidas entre los 6 y los 11 años.
}

\section{BIBLIOGRAFÍA.}

AREA MOREIRA, M. Tecnologías digitales, multialfabetización y bibliotecas en la escuela del siglo XXI. Boletín de la Asociación Andaluza de Bibliotecarios [en línea], 2010, $\mathrm{n}^{\mathrm{o}}$ 98-99, p. 39-52. Disponible en internet: $<$ http://www.aab.es/aab/images/stories/Boletin/98_99/2.pdf $>$. [Consultado: 11 de agosto de 2012].

BARÓ, M. y MAÑ̀̀, T. Formarse para informarse. Madrid: Celeste, 1996.

BERNERS-LEE, T. Tejiendo la red: el inventor de la World Wide Web nos descubre su origen. Madrid: Siglo XXI, 2000.

Las Bibliotecas escolares en España: análisis y recomendaciones. Dirección, Álvaro Marchesi, Inés Miret. Salamanca: Fundación Germán Sánchez Ruipérez, 2005.

CAMACHO ESPINOSA, J.A. La biblioteca escolar en España: pasado, presente... y un modelo para el futuro. Madrid: Ediciones de la Torre, 2004.

CASSANY, D. y AYALA, G. Nativos e inmigrantes digitales en la escuela. Participación Educativa [en línea], 2008, $\mathrm{n}^{\mathrm{0}}$ 9, noviembre, p. 53-71. Disponible en internet: $<$ http://www.fapaes.net/pdf/web_participacion_educativa_09.pdf $>$. [Consultado: $10 \mathrm{de}$ agosto de 2012].

CASTÁN LANASPA, G. Bibliotecas escolares y profesores: las dificultades de un idilio en ciernes. Mi Biblioteca: la revista del mundo bibliotecario, 2006, año II, $\mathrm{n}^{\mathrm{o}}$ 7, otoño, p. 70-73.

CASTÁN LANASPA, G. Concepto, objetivos y funciones de la biblioteca escolar. En Guía para bibliotecas escolares, Pedro López Gómez, José Carlos Santos Paz (eds.), A Coruña: Universidade da Coruña, 2009, p. 25-50.

CASTELLS, M. La galaxia Internet: reflexiones sobre Internet, empresa y sociedad. Barcelona: Areté, 2001.

CASTILLO DÍAZ. A. La comunicación de las marcas universitarias españolas a través de sus sedes webs institucionales. Tesis doctoral. Universidad de Extremadura, 2007.

CENCERRADO MALMIERCA, L.M. y EGIDO DE ARRIBA, R. Directorio de Bibliotecas Escolares Españolas, Fundación Germán Sánchez Ruipérez. En Anuario de Bibliotecas Españolas 2010, Conchi Jiménez Fernández (coord.). Málaga: Fundación Alonso Quijano, 2010, p. 125-129. 
CERVERO GUERRERO, J. Proyecto de innovación educativa. Disponible en: $<$ http://sites.google.com/site/fuentenuevaes/planes-y-proyectos-educativos/proyectode-innovacion-educativa $>$. [Consultado: 12 de septiembre de 2012].

CODINA, L. Evaluación de recursos digitales en línea: conceptos, indicadores y métodos. Revista Española de Documentación Científica, 2000, vol. 23, nº 1, p. 9-44.

Directrices de la IFLA/UNESCO para la biblioteca escolar [en línea]. 2002, Disponible en internet: < http://archive.ifla.org/VII/s11/pubs/sguide02-s.pdf>. [Consultado: 29 de agosto de 2012].

DURBAN ROCA, G. Tensiones y distensiones. Reflexión en torno a la situación actual de la biblioteca escolar. Libro Abierto: Bibliotecas escolares de la provincia de Málaga: Boletín de información y apoyo, 2009, $\mathrm{n}^{\circ} 35$, febrero, p. 6-11.

DURBAN ROCA, G.; CID PROLONGO, A. y GARCÍA GUERRERO, J. Programas para el desarrollo de la competencia informacional articulados desde la biblioteca escolar, Documento de Referencia para bibliotecas escolares DR4/BECREA. Sevilla: Junta de Andalucía, Consejería de Educación, 2012.

ENSIL. European Network for School Libraries and Information Literacy [en línea]. Disponible en internet: <http://www.skolbibliotek.se/pdf/ensilfolder.pdf $>$. [Consultado: 16 de agosto de 2012].

ESPAÑA. Real Decreto 1513/2006, de 7 de diciembre, por el que se establecen las enseñanzas mínimas de la Educación Primaria. Boletín Oficial del Estado, no 293, 8 de diciembre, p. 43053-43102.

Estándares para las bibliotecas escolares CRA. Santiago de Chile: Ministerio de Educación de Chile, 2011.

GARCÍA ANDRÉS, M.A. Leer y escribir en la era de Internet: Análisis y propuestas para la lectura y la escritura en Secundaria. Pamplona: Gobierno de Navarra, Departamento de Educación, 2008.

GARCÍA GUERRO, J. y LUQUE JAIME, J.M. Evaluación Bibliotecas Escolares CREA, provincia de Málaga, curso 2010/2011. Libro Abierto de información y apoyo a las bibliotecas escolares de la provincia de Málaga [en línea]. 2011. Disponible en internet:

$<$ http://www.juntadeandalucia.es/averroes/bibliotecaescolar/images/MisPdf/separatas/1 ibroA45-sep11 separata.pdf.pdf>. [Consultado: 8 de enero de 2012].

GARCÍA GUERRERO, J. y LUQUE JAIME, J.M. Organización y funcionamiento de la biblioteca escolar: tareas básicas, Documento de Referencia para bibliotecas escolares DR2/BECREA [en línea]. Sevilla: Junta de Andalucía, Consejería de Educación, 2011. Disponible en internet: $<$ http://www.juntadeandalucia.es/averroes/bibliotecaescolar/images/MisPdf/DR2/DR2 BECREA.pdf $>$. [Consultado: 21 de enero de 2012].

GARCÍA GUERRERO, J. Contribución de la biblioteca escolar al formato de la lectura, Documento de Referencia para bibliotecas escolares DR3/BECREA. Sevilla: Junta de Andalucía, Consejería de Educación, 2012.

GÓMEZ HERNÁNDEZ, J.A. Alfabetización informacional: cuestiones básicas. Anuario ThinkEPI, 2007, p. 43-50. 
GÓMEZ HERNÁNDEZ, J.A. Las bibliotecas escolares en España ante una nueva década", Anuario ThinkEPI, 2010, p. 94-102.

HEIMLICH, J.E. y WANG, K. Evaluating the structure of web sites. Enviromental Education and Training Partnership (EETAP) Resource Library [en línea]. Columbus, Ohio: The Ohio State University, 1999. Disponible en internet: $<$ http://www.epa.gov/enviroed/pdf/evalstruc.pdf $>$. [Consultado: 26 de diciembre de 2011].

I Congreso Nacional de Bibliotecas Escolares: Resumen y conclusiones. Salamanca: Fundación Germán Sánchez Ruipérez, 2006.

I Encuentro Nacional sobre bibliotecas escolares: Conclusiones: Madrid, 13, 14 y 15 de marzo. Educación y Biblioteca, 1997, año 9, n 78, abril, p. 10-23.

JIMÉNEZ PIANO, M. y ORTIZ-REPISO JIMÉNEZ, V. Evaluación y calidad de sedes web. Gijón: Trea, 2007.

LARA ESCOZ, J.I. Posibilidades y proyección de la biblioteca escolar en las redes sociales y en la web 2.0. En Blog Libro Abierto [en línea]. 2011. Disponible en internet: $<$ http://cepronda.org/libroabierto/?p=1814>. [Consultado: 27 de diciembre de $2011]$.

LUQUE JAIME, J.M. Servicios y programas de la biblioteca escolar en las páginas webs de los centros educativos. Selección de recursos digitales y virtuales de apoyo al currículo. En GARCÍA GUERRERO, J. (dir.). La articulación de los recursos en el funcionamiento de la biblioteca escolar. Madrid: Ministerio de Educación y Ciencia, Secretaría General de Educación, 2007, p. 181-220.

Manifiesto de la IFLA/UNESCO sobre Internet [en línea]. 2006. Disponible en internet: $<$ http://www.ifla.org/publications/the-ifla-internet-manifesto $>$. [Consultado: $11 \mathrm{de}$ octubre de 2011].

Manifiesto UNESCO/IFLA sobre la Biblioteca Escolar [en línea]. 2000. Disponible en internet:

$<$ http://www.unesco.org/webworld/libraries/manifestos/school_manifesto_es.html>. [Consultado: 8 de octubre de 2011].

Marco de referencia para las bibliotecas escolares [en línea]. Madrid: Ministerio de Educación, 2011 . Dadrid, en internet: $<$ http://www.mcu.es/bibliotecas/docs/MC/ConsejoCb/CTC/Marcoreferenciabescolares .pdf $>$. [Consultado: 25 de diciembre de 2011].

MERLO VEGA, J.A. La evaluación de la calidad de la información web: aportaciones teóricas y experiencias prácticas [en línea]. En ZAPICO ALONSO, F. (coord.). Recursos informativos: creación, descripción y evaluación. Mérida: Junta de Extremadura, 2003, p. 101-110. (Sociedad de la información, 8). Disponible en: $<$ http://exlibris.usal.es/merlo/escritos/pdf/calidad.pdf $>$. [Consultado: 9 de septiembre de 2011].

MERLO VEGA, J.A. Información y referencia en entornos digitales: desarrollo de servicios bibliotecarios de consulta. Murcia: Universidad de Murcia, 2009.

MIRET, I.; BARÓ, M.; MAÑÀ, T.; VELLOSILLO, I. y MONTERO, I. Bibliotecas escolares «entre comillas». Madrid: Fundación Germán Sánchez Ruipérez; Ministerio de Educación, 2010. 
MIRET, I.; BARÓ, M.; MAÑÀ, T. y VELLOSILLO, I. Bibliotecas escolares ¿entre interrogantes? Madrid: Fundación Germán Sánchez Ruipérez; Ministerio de Educación, 2011.

MONGUILOT, I. La autoevaluación de la biblioteca escolar debe ser asumida por el centro. En Bibliotecas Escolares ¿entre interrogantes?: Herramienta de autoevaluación: preguntas e indicadores para mejorar la biblioteca [en línea]. Madrid: Ministerio de Educación, 2011, p. 143-144. Disponible en internet: $<$ http://www.lecturalab.org/uploads/website/docs/2684-2-

Bibliotecas_escolares_entre_interrogantes.pdf $>$. [Consultado: 13 de enero de 2012].

MURPHY, R. Going Online: Devoloping LRC web page. Swindon (Inglaterra): School Library Association, 2003.

REITZ, J.M. ODLIS: Online Dictionary for Library and Information Science [en línea]. 2004. Disponible en internet: <http://www.abc-clio.com/ODLIS/odlis_A.aspx>. [Consultado: 24 de diciembre de 2011].

ROMAGNOLI, C.; FEMENÍAS, G. y CONTE, P. Internet, un nuevo recurso para la educación. Material de apoyo para profesores. Santiago de Chile: Ministerio de Educación, 2001.

School Libraries and Resource Centers Section [en línea]. IFLA: International Federation of Library Associations and Institutions, 2012. Disponible en internet: $<$ http://www.ifla.org/en/about-school-libraries-resource-centers $>$. [Consultado: 13 de agosto de 2011].

Teaching Reading in Europe: Contexts, Policies and Practices [en línea]. Education, Audiovisual and Culture Executive Agency, P9 Eurydice, Brussels, 2011. Disponible en internet: $<$ http://eacea.ec.europa.eu/education/eurydice/documents/thematic_reports/130EN.pdf >. [Consultado: 10 de enero de 2012].

The Mission of IASL [en línea]. IASL: International Association of School Librarianship, 2007. Disponible en internet: $<\mathrm{http}: / / \mathrm{www}$.iasl-online.org/about $>$. [Consultado: $15 \mathrm{de}$ enero de 2012].

VIVANCOS, J. Tratamiento de la información y competencia digital. Madrid: Alianza Editorial, 2008.

YÁGUEZ, E. Guía práctica para el desarrollo y dinamización de la Biblioteca Escolar en Secundaria. Madrid: Ministerio de Educación, 2006. 